\title{
Bioanalysis
}

\section{Metabonomic diagnosis of bladder cancer: what are the advantages and considerations?}

\author{
"Metabonomics demonstrates great potential in characterizing bladder cancer, \\ identification of marker metabolites and shedding insights into the pathogenesis \\ and potential therapeutic targets of the disease."
}

Keywords: advantages $\bullet$ bladder cancer $\bullet$ considerations $\bullet$ diagnosis $\bullet$ metabonomics

Bladder cancer (BC) is the fifth most common cancer and is one of the leading causes of death worldwide [1]. The 5-year survival rate for BC is approximately $94 \%$ if detected early; thus timely diagnosis and intervention of BC increase patient's survival rate dramatically [2]. However, BC has a high recurrence rate and patients are subjected to life-long surveillance. As a result, the lifetime economic burden per BC patient is higher than other cancer types. Currently, the standard diagnoses of BC are cystoscopy and urinary cytology [3]. While cystoscopy is clinically reliable, it is expensive, invasive and associated with a definite risk of morbidity. On the other hand, noninvasive urinary cytology demonstrates poor sensitivity in detecting low-grade BC (4-31\%) [4]. In recent years, several proteinaceous urinebased bladder tumor markers (UBBTMs) have been evaluated for the diagnosis of BC, such as bladder tumor antigen (BTA), NMP22, FDP and ImmunoCyt [5]. However, the specificity and sensitivity of current molecular biomarkers are not adequate to replace cystoscopy [6]. Therefore, there is an impetus to develop sensitive and specific noninvasive biomarkers for the diagnosis and surveillance of BC.

Dysregulated molecular pathways, associated with tumor genes, secrete specific modulated levels of metabolites into biological fluids that may be detectable prior to clinical symptoms, rendering them potential early biomarkers for cancer diagnosis. Metabonomics measures the dynamic multiparametric responses of systems biological metabolome to genetic modifications or pathophysiological stimuli such as cancers [7]. It allows scientists to survey global dysregulated metabolic pathways and gain holistic insights into cancer etiology and progression. Significant progress has been made using modern metabonomics techniques to characterize BC patients, identify marker metabolites and generate new knowledge in disease biology and potential therapeutic targets [5]

\section{Advantages}

The application of metabonomics in the diagnosis of BC is particularly intriguing due to the possibility of using urine for detecting malignancy and determining prognosis. As urine is bathed in close anatomical proximity to the bladder carcinoma, it is expectedly a suitable diagnostic fluid. Advantages of urinary metabonomics profiling of BC include noninvasive sample collection, relative ease of urine analysis, low-cost, circumvention of subjective pathological assessment and urinary metabolic phenotype (metabotype) that is sensitive to environmental, gut microbiota and host genetic factors.

Most current BC metabonomics studies showed that BC patients could be distinctly differentiated from healthy controls with high sensitivity (>90\%) and specificity (>90\%) [8-14]. A number of potential marker metabolites of $\mathrm{BC}$ are derived from metabolic pathways associated with glycolysis, glutathione, tryptophan, purine, pyrimidine, carnitine, citrate, taurine and hippuric acid [5]. Such dysregulated marker metabolites are useful in deciphering the BC biology and have the potential to be translated
Yanjun Hong

Department of Pharmacy, Faculty of Science, National University of Singapore, 18 Science Drive 4, 117543 Singapore

Kesavan Esuvaranathan Department of Surgery, National University Health System, 5 Lower Kent Ridge Road, 119074 Singapore

Kishore Kumar Pasikanti Department of Pharmacy, Faculty of Science, National University of Singapore, 18 Science Drive 4, 117543 Singapore

Eric Chun Yong Chan

Author for correspondence:

Department of Pharmacy, Faculty of

Science, National University of Singapore, 18 Science Drive 4, 117543 Singapore

Tel.: +65 65166137

Fax: +6567791554

phaccye@nus.edu.sg
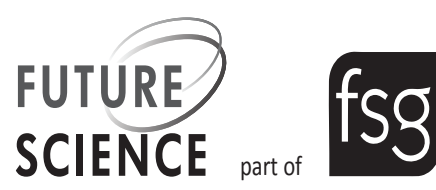
to clinical applications upon further evaluation and validation.

In addition to accurate diagnosis, classification of bladder tumors is important for prognosis of BC. However, reproducibility and consistency in defining superficial bladder tumors is a chronic challenge for pathologists. Notably, Cao et al. demonstrated that distinct metabotypes were associated with lowgrade versus high-grade $\mathrm{BC}$ as well as pre- versus posttransurethral resected cohorts [15]. Additionally, two $\mathrm{BC}$ metabonomics studies exhibited potential in the staging and grading of bladder tumors [8,13]. Based on the direct correlation of metabolic features with pathological status, this technique will provide a mechanistic handle for probing the progression of $\mathrm{BC}$.

\section{"Most current bladder cancer metabonomics studies showed that bladder cancer patients could be distinctly differentiated from healthy controls with high sensitivity $(>90 \%)$ and specificity (>90\%)."}

Monitoring treatment and recurrence is critical for BC patients. Recently, Jin et al. demonstrated in a large cohort study (138 BC and 121 healthy controls) that $\mathrm{BC}$ metabotype correlates positively with cancerspecific survival time, which in turn underscores the possibility of predicting postdiagnosis survival time of $\mathrm{BC}$ patients via metabonomics [14].

As both bladder and kidney are in direct contact with urine, it is possible that marker metabolites associated with $\mathrm{BC}$ and kidney cancer are excreted into urine. Intriguingly, it has been reported that metabonomics can discriminate BC and kidney cancer successfully, showing $100 \%$ sensitivity and specificity [13].

\section{Considerations}

Although metabonomics is a promising approach in the diagnosis and surveillance of $\mathrm{BC}$, several intrinsic limitations still exist. Four key considerations are summarized below.

- The number of patients recruited in most BC metabonomics studies was relatively inadequate. Although external validation was performed in most of the current BC metabonomics studies, the validation cohorts were equally small. Therefore, further validation of potential BC biomarkers should be based on a statistically powerful clinical sample size. To achieve this objective, it becomes pertinent for clinician scientists to conduct multicenter collaborative clinical trials.

- For most current BC metabonomics studies, only healthy controls and $\mathrm{BC}$ patients were examined.
BC patients are typically presented with hematuria, a nonspecific symptom that can become a serious confounding variable. Other concomitant conditions such as kidney cancer and urinary tract infection or inflammation may also modulate the metabotype. Therefore, to better validate the specificity and sensitivity of $\mathrm{BC}$ biomarkers, a more diverse patient population is essential and should be carefully enrolled in future clinical trial.

- There is relatively little overlap between the potential biomarkers identified in individual $\mathrm{BC}$ metabonomics studies [5]. Metabotype is highly dynamic and sensitive to environmental factors (e.g., sample storage conditions) [16], host factors (e.g., age, disease, gender, genetic) and external factors (e.g., diet, smoking, chronic medications) [17]. Additionally, the metabotypes derived from different analytical platforms are variable due to differential chemical space coverage. Therefore, standardization of the metabonomics experiment (e.g., clinical trial design and selection of metabonomics techniques) is critical to ensure good-quality data.

- Global metabonomics are semi-quantitative in measuring biomarkers. However, most studies did not quantitate the potential biomarkers using external sample set, which might be due to the fact that marker metabolite quantitation is difficult and time-consuming, especially if the standard metabolite is not commercially available and has to be chemically synthesized. Despite these challenges, validation of important marker metabolites using quantitative methods remains critical.

\section{Conclusion \& future perspective}

Early diagnosis and life-long surveillance are clinically important to improve the long-term survival of BC patients. Currently, a noninvasive biomarker with high sensitivity and specificity in detecting bladder tumors is lacking. Metabonomics enables mapping of early biochemical changes in disease and development of predictive biomarkers. Metabonomics demonstrates great potential in characterizing $\mathrm{BC}$, identification of marker metabolites and shedding insights into the pathogenesis and potential therapeutic targets of the disease. In the future, metabonomics may be potentially explored for the holistic clinical management of $\mathrm{BC}$ patients from diagnosis to intervention. For instance, rapid evaporation ionization mass spectrometry (REIMS) has been applied for intraoperative analysis of tumor specimens in vivo [18]. REIMS intelligent knife (REIMS-iKnife) was shown to be able to differentiate accurately between distinct histological and histopathological tissue types. Such a metabonomics-inspired technology is poten- 
tially applicable for the surgical intervention of $\mathrm{BC}$ in the future. Notably, near-real-time identification of positive resection margins could help improve the functional outcomes of the bladder, by mitigating surgical trauma and reducing the removal of healthy tissues.

\section{Financial \& competing interests disclosure}

The research was supported by the Singapore Ministry of Health's National Medical Research Council under its Individual

\section{References}

1 Siegel R, Ward E, Brawley O, Jemal A. Cancer statistics, 2011. CA: Cancer J. Clin. 61(4), 212-236 (2011).

2 Urquidi V, Rosser CJ, Goodison S. Molecular diagnostic trends in urological cancer: biomarkers for non-invasive diagnosis. Curr. Med. Chem. 19(22), 3653-3663 (2012).

3 Mitra AP, Cote RJ. Molecular screening for bladder cancer: progress and potential. Nat. Rev. Urol. 7(1), 11-20 (2010).

4 Lotan Y, Roehrborn CG. Sensitivity and specificity of commonly available bladder tumor markers versus cytology: results of a comprehensive literature review and meta-analyses. Urology 61(1), 109-118 (2003).

5 Chan EC, Pasikanti KK, Hong Y et al. Metabonomic profiling of bladder cancer. J. Proteome Res. 14(2), 587-602 (2015).

6 Robinson VL, Porter M, Messing E et al. BCAN Think Tank session 2: Molecular detection of bladder cancer: the path to progress. Urol. Oncol. 28(3), 334-337 (2010).

7 Nicholson JK, Lindon JC, Holmes E. 'Metabonomics': understanding the metabolic responses of living systems to pathophysiological stimuli via multivariate statistical analysis of biological NMR spectroscopic data. Xenobiotica 29(11), 1181-1189 (1999).

8 Pasikanti KK, Esuvaranathan K, Ho PC et al. Noninvasive urinary metabonomic diagnosis of human bladder cancer. J. Proteome Res. 9(6), 2988-2995 (2010).

9 Bansal N, Gupta A, Mitash N et al. Low-and high-grade bladder cancer determination via human serum-based metabolomics approach. J. Proteome Res. 12(12), 5839-5850 (2013).
Research Grant scheme (R-176-000-119-213 to K Esuvaranathan and ECY Chan). KK Pasikanti was a recipient of the National University of Singapore President Graduate Fellowship. The authors have no other relevant affiliations or financial involvement with any organization or entity with a financial interest in or financial conflict with the subject matter or materials discussed in the manuscript apart from those disclosed.

No writing assistance was utilized in the production of this manuscript.

10 Pasikanti KK, Esuvaranathan K, Hong Y et al. Urinary metabotyping of bladder cancer using two-dimensional gas chromatography time-of-flight mass spectrometry. J. Proteome Res. 12(9), 3865-3873 (2013).

11 Issaq $\mathrm{H}$, Nativ $\mathrm{O}$, Waybright $\mathrm{T}$ et al. Detection of bladder cancer in human urine by metabolomic profiling using high performance liquid chromatography/mass spectrometry. J. Urol. 179(6), 2422-2426 (2008).

12 Huang Z, Lin L, Gao Y et al. Bladder cancer determination via two urinary metabolites: a biomarker pattern approach. Mol. Cell. Proteomics 10(10), M111. 007922 (2011).

13 Huang Z, Chen Y, Hang W et al. Holistic metabonomic profiling of urine affords potential early diagnosis for bladder and kidney cancers. Metabolomics 9(1), 119-129 (2013).

14 Jin X, Yun SJ, Jeong P, Kim IY, Kim WJ, Park S. Diagnosis of bladder cancer and prediction of survival by urinary metabolomics. Oncotarget 5(6), 1635-1645 (2014).

15 Cao M, Zhao L, Chen H, Xue W, Lin D. NMR-based metabolomic analysis of human bladder cancer. Anal. Sci. 28(5), 451-456 (2012).

16 Monteiro M, Carvalho M, Bastos M, Guedes de Pinho P. Metabolomics analysis for biomarker discovery: advances and challenges. Curr. Med. Chem. 20(2), 257-271 (2013).

17 Weiss RH, Kim K. Metabolomics in the study of kidney diseases. Nat. Rev. Neurol. 8(1), 22-33 (2012).

18 Balog J, Sasi-Szabó L, Kinross J. Intraoperative tissue identification using rapid evaporative ionization mass spectrometry. Sci. Transl. Med. 5(194), $194 \mathrm{ra} 93$ (2013). 